\title{
Control of drying by means of IR detection of thermal waves
}

\author{
by I. Delgadillo-Holtfort ${ }^{1}$, J. Pelzl, and B.K. Bein
}

AG Solid State Spectroscopy, Exp.-Phys. III, Ruhr-University, D-44780 Bochum, Germany ${ }^{1}$ on leave from Depto. Física CINVESTAV - IPN, México D.F., E-mail: delgadillo@ep3.ruhr-uni-bochum.de

\begin{abstract}
Frequency-dependent thermal wave measurements based on IR detection have been used to study drying processes in different types of materials, namely foodstuff, construction materials and textiles. It has been found that this technique can give information on the total moisture content, on the moisture depth distribution, and on the progress of drying with time.
\end{abstract}

\section{Introduction}

Drying processes and the measurement of the moisture content are of basic importance in many industrial sectors and materials. For example in the textiles industry, finishing processes consist of sequences of wetting processes followed by drying to remove the solvents. In the development and design of new products in the textiles industry, on the other hand, information about the moisture accumulation can contribute to improve the comfort of functional textiles. In civil engineering, the localization and quantification of moisture in walls and construction materials can serve to reduce costs in draining and reconstruction and can be decisive in technical reports and lawsuits. In the production of foodstuff, drying or the reduction of drying by means of packaging foils are relevant for the quality of foodstuff materials.

In the present work we explore the possibility to control drying processes and to determine the moisture content and its depth distribution in different types of materials (construction materials, textiles, foodstuff materials) by means of thermal wave measurements using non-contact remote IR detection. These measurements and their interpretation are based on the fact that in porous materials the effective thermal transport properties can change considerably, if the pore space, containing air in the dry material, is partially filled with water or water vapor. The water content contributes to establish a continuous phase of solid/liquid material of generally higher thermal conductivity and effusivity and the heat diffusion can additionally be supported by the diffusion of water vapor.

In Section 2, the principles of IR detection of thermal waves are very briefly recalled, and in Section 3 the used measurement device is presented, as well as the handling of the different wet and dry samples. The various results are presented and discussed in Section 4, followed by the conclusions in Section 5 .

\section{Measurement principles}

Thermal waves, i.e., time- and space-dependent temperature distributions, can be excited through intensity-modulated surface heating. Such wave-like temperature oscillations depend on the thermophysical parameters relevant for time-dependent heating processes, namely the thermal diffusivity $\alpha$ and the effusivity $e$. These waves are of diffusive nature and are characterized by the so-called thermal diffusion length given by $\mu=\sqrt{\alpha / \pi f}$. Since it varies with the modulation frequency $f$ of the heating, the penetration depth of thermal waves $x \propto \mu$ can be varied by a variation of the modulation frequency, enabling depthdependent measurements of the effective thermal properties.

Thermal waves excitation obtained through absorption of intensity-modulated electromagnetic radiation at the surface of the body and detection of the IR radiation emitted by the 
sample's surface is an absolutely non-contact remote method, useful for measuring the effective thermal properties of arbitrary bodies. Since on the other hand, in porous bodies the thermal properties can vary considerably with the moisture content stored in the pore space, the thermal wave method should also be very efficient for non-contact remote measurements of the moisture content. From soil materials, it is known that the effusivity is the property that mainly changes with the moisture content [1], whereas the thermal diffusivity remains nearly constant. This agrees with the fact that the effects of moisture $F$ and porosity $p$ on thermal conductivity $k$, mass density $\rho$, and specific heat capacity $c$ are accumulated in the effusivity,

$$
e=\sqrt{k(p, F) \rho(p, F) c(p, F)} \quad,
$$

whereas they partially eliminate in the thermal diffusivity

$$
\alpha=\frac{k(p, F)}{\rho(p, F) c(p, F)} \approx \alpha_{0}
$$

The influence of the technical parameters and frequency-characteristics of the detection system can be eliminated in thermal wave measurements by measurements of reference samples of known thermal and optical properties, e.g. a sample of glassy carbon. In the application of IR radiometry of thermal waves to moisture measurements, the measurement of the corresponding dry samples provides an alternative reference. Since the modulated radiation signal is proportional to the thermal wave and since this latter one is inversely proportional to the effective effusivity, we have that

$$
S(f) \propto \varepsilon \delta T_{s}(f) \propto \frac{\varepsilon_{s} \eta_{s}}{e_{s}}
$$

with $\varepsilon$ being the effective emissivity, and $\eta$ the photothermal efficiency. The inversely normalized amplitudes of dry and wet samples of the same material

$$
S_{n}{ }^{-1}\left(f^{-1 / 2}\right)=\frac{S_{d r y}(f)}{S_{w e t}(f)} \propto \frac{\varepsilon_{d r y} \delta T_{d r y}(f)}{\varepsilon_{w e t} \delta T_{w e t}(f)} \propto \frac{\varepsilon_{d r y} \eta_{d r y}}{e_{d r y}} \cdot \frac{e_{w e t}}{\varepsilon_{w e t} \eta_{w e t}}=\frac{\varepsilon_{d r y} \eta_{d r y}}{\varepsilon_{w e t} \eta_{w e t}} \cdot \frac{e_{w e t}}{e_{d r y}}
$$

plotted versus the square root of the modulation frequency, can therefore supply direct information on the moisture content and its profile. In first approximation one can assume that the photothermal efficiency is independent of the moisture content. Depending on the dominance of the moisture content on the effective emissivity or alternatively on the thermal effusivity, the inversely normalized amplitudes can decrease or increase with the moisture content. The depth profile resulting from inverse normalization according to (4) can mainly be considered as an effusivity depth profile, once that the emissivity and the photothermal efficiency are mainly optical properties of the surface.

\section{Experimental}

Photothermal measurements based on modulated heating in the visible spectral range and IR detection of the thermal wave response are used here in a reflection configuration in which the heated sample surface is the same facing the IR detector. Our measuring system consists of three main components: an Argon ion laser (Spectra Physics, Series 2000) to excite thermal waves of small temperature amplitude, an IR detector to measure the emitted radiation and a Lock-in amplifier to filter the small periodical variations of IR emission, corresponding to the thermal wave $\delta T(f)$ from the comparatively large radiation background corresponding to the stationary sample temperature.

For the periodical heating of the samples the beam of the Ar ion laser has been used at the wavelength of $514 \mathrm{~nm}$. The laser beam has been modulated with the help of an acoustooptical modulator. For the detection of the IR radiation, a nitrogen-cooled photoconducting $\mathrm{HgCdTe}$ detector has been used, together with IR optics consisting of two $\mathrm{BaF}_{2}$ lenses and a Ge cut-on filter to eliminate possible contributions from scattered visible light. The detectable 
wavelength interval is limited by $1 \mu \mathrm{m}<\lambda<12 \mu \mathrm{m}$. Details of the measuring system have been described elsewhere [2].

In general, the photothermal measurements have first been done for the dry and then for wet samples. For the control of the water content, weighing before and after each photothermal measurement has been done, and for the purpose of normalization and comparison of the different measurements, photothermal signals of glassy carbon have repeatedly been measured as general reference measurement. In order to reduce the influence of laser beam heating on the samples' water content, preliminary measurements have been done, reducing the power per surface area by large heating spots and an effective low laser power.

The construction materials studied include artificial lime sand-stone, stucco, cement mortar, concrete, and brick-stone. Wet samples have been obtained by soaking them in water for several days and then they have been exposed to the atmosphere to simulate natural drying processes.

The textiles studied are white and colored knitted fabrics consisting of one-yarn and twoyarns (cotton and polyamide) per stitch. Due to this construction, the two-yarns per stitch fabric exposes polyamide at one surface and cotton at the other one. It has been measured at both sides, both in dry and different wet states. The textile had previously been dried during 12 hours at $105^{\circ} \mathrm{C}$.

Two types of foodstuff have been analyzed, Dutch cheese "young Gouda" and a German Rotwurst, "Pomeranian kind". The two products had been bought in an ordinary supermarket in the usual plastic foil - wrapped presentation.

Young Gouda is a compact mass known for its high moisture content, and thus we expected to find measurable effects already after relatively short drying times. A fresh cut from the interior of the packed-up piece of cheese was first measured and then the evolution of various surface cuts with time was measured repeatedly. During this time, bacteriological spoilage due to microorganisms could not be not be observed by sight.

The measured sausage consists of a mixture of ingredients (blood, pieces of liver, tongue, and bacon, several spices and ascorbic acid as conservation stuff). The sausage had got a skin of natural swain intestine and had been smoked-dried. Measurements have been done for two sausage regions showing homogeneous but different aspects of the skin: at the first position the skin was relatively thin and transparent, at the other it was relatively thick and white. The process of drying in the atmosphere under natural conditions, after taking the sausage out of the plastics foil, was accompanied by photothermal measurements.

\section{Results}

In Fig. 1 we show the signal amplitudes measured for (a) artificial lime sand-stone for both the dry (a) and a wet (a) state, and (b) for the polyamide surface of a two-yarns per stitch fabric at different moisture contents. In both figures, the signals measured for glassy carbon (x) are shown for comparison, and in Fig. $1 \mathrm{~b}$ the noise signal is shown, illustrating the reliability of our measurements. As can be seen for the two materials, the signals vary markedly with the moisture content. Moreover, the signal amplitudes decrease with increasing moisture content, which means that the ratio emissivity-to-effusivity cle decreases with increasing moisture content. This means, even if the emissivity increases with growing moisture content, the increase of the thermal effusivity with the moisture content is clearly dominant.

\subsection{Depth profiling}

In Fig. 2 we show the inverse normalized signals of several construction materials, all of them at $12 \%$ moisture content, represented as function of the quantity $1 / \sqrt{f}$ that is directly 
proportional to the penetration depth. In this case, the signals measured for the corresponding dry materials were used as reference. Thus, the inverse normalized amplitudes give, according to (4), a direct impression of the depth distribution of moisture in the samples. The comparison of the different construction materials clearly shows that artificial lime-sandstone (*), stucco $(\Delta)$ and cement mortar (o) store the moisture just at the surface, according to the much larger values at higher frequencies, corresponding to small penetration depths. In contrast, in brick-stone ( $\mathbf{a})$ the moisture is accumulated in the bulk of the samples, at lower frequencies corresponding to larger penetration depths. This effect, keeping the moisture far away from the surface and atmosphere where we live, contributes to the comfort of brick-stone houses [3].

Also interested in comfortable feeling is the textile industry, in which the design of fabrics that keep the moisture away from the human body is very important. In Fig. 3, the depth profiles of moisture obtained during the progress of drying for a two-yarns per stitch fabric are shown. In Fig. 3a the moisture depth profiles are shown from the polyamide side and in Fig. $3 \mathrm{~b}$ from the cotton side of the two-yarns/stitch fabric. For these normalized amplitudes the signals measured for the corresponding dry sides of the textile have been used as reference. In Fig. 3a we can see that the moisture depth distribution with increasing penetration depth $x \propto 1 / \sqrt{f}$ seems to be nearly constant, only with a slight increase for the lower moisture contents $(119 \%, 52 \%$ and 10\%) at the larger penetration depths. This means, the moisture is preferentially stored deeper below the polyamide surface, namely in the cotton fibers of the two-yarns per stitch fabric. In Fig. 3b in contrast, we can see that the moisture depth distribution with increasing penetration depth seems to be nearly constant only for the low moisture contents (23\%, $8 \%)$. For the higher moisture contents (156\%, 85 $\%)$, the moisture effects are accumulated at the smaller penetration depths, which means the moisture is preferentially accumulated in the cotton region of the two-yarns/stitch fabric.

The dry-to-wet normalization procedure used above for textiles or construction materials is very useful, as long as drying is the only process involved in the changing properties and as long as there is a unique relation between the moisture content and the effective thermal properties.

For cases in which additional processes may be involved, the comparison of the "dry" and "wet" state may not be directly accessible to interpretation as the additional processes are not considered. This case is shown in Fig. 4, in which the amplitudes of drying Gouda cheese samples are normalized with respect to the amplitude of the fresh surface cut. Three phases of drying can be identified from these measurements: (i) during the first 30 minutes of drying, the effusivity decreases in a layer of growing thickness, an effect probably due to the loss of water at the surface; (ii) During the second phase (up to $270 \mathrm{~min}$ ) the thickness of the dry surface layer continues to increase whereas the inversely normalized signals just at the surface show a reverse effect, increasing again. This may be associated to changes of the optical properties at the surface, namely the formation of a translucent crust; (iii) During the third phase, after $300 \mathrm{~min}$, the values $S_{n}^{-1}(f)$ at the very surface start to decrease again, showing a second reverse effect which may be associated to sweating out of fat.

As for young Gouda cheese, studies of the progress of drying or ripening are of interest for other foodstuffs. This is for instance the case in our studies performed with a German "Rotwurst", where the information about the progress of drying had to be obtained from the signal phases rather than from the amplitudes of the photothermal signals. The normalized phases for the thinner dark skin region are shown in Fig. $5 \mathrm{a}$, whereas the phases of the thicker white skin region are shown in Fig. 5b. For both cases, and in contrast with the former results of this paper, the reference material used is glassy carbon. In the two cases, the phases are plotted in the penetration depth proportional representation $1 / \sqrt{f}$. Two main common features have to be noted for both series of measurements: Namely, the existence of two frequency (depth) regions in which the signals behave markedly different. At the high frequency region -below $1 / \sqrt{f} \approx 0.18 \sqrt{\mathrm{Hz}}$ and below $1 / \sqrt{f} \approx 0.4 \sqrt{\mathrm{Hz}}$ for the thin and the 
thicker sausage skin regions respectively-, i.e., in the skin region the phases show rather small changes. In contrast, at low frequencies, i.e., within the sausage material they show a rising tendency with the progress of time and drying.

\section{Conclusions}

We have been able to detect changes in water content in textiles, construction materials and foodstuff by monitoring the changes in the samples' effective thermal properties by means of IR radiometry of thermal waves. We have found that the measured frequencydependent IR signals vary markedly with the moisture content, and that the main effect is due to changes of the thermal effusivity. By using the corresponding dry samples as reference it is possible to visualize the moisture depth distribution directly.

The advantage of IR detection is that it works non-contact, in remote detection, and that it thus can be applied under industrial conditions.

\section{References}

[1] B.K. Bein, W. Kiepert, H.J. Obramski, C. Gruss, J. Bolte, J. Pelzl, P. Jütterschenke, H.J. Blonsky, W. Karsch, J. Nitsch, Thermophysical Aspects of Soil Decontamination by Dielectric Heating", High Temp.- High Pressures 29, 1997, 431-442.

[2] J. Bolte, B.K. Bein, J. Pelzl, IR Detection of Thermal Waves - Effect of Imaging Conditions on the Background Fluctuation Limit, In: Quantitative InfraRed Thermography QIRT'96, eds. D. Balageas, G. Busse a. C.M. Carlomagno, Eurotherm Series 50, Edizioni ETS, Pisa, 1997, 9-14.

[3] B.K. Bein, I. Delgadillo-Holtfort, J. Gibkes, J. Pelzl, Photothermische Feuchtebestimmung an Baumaterialien, BAM - DGZfP-Berichtsband BB 69-CD, 1999, M1.

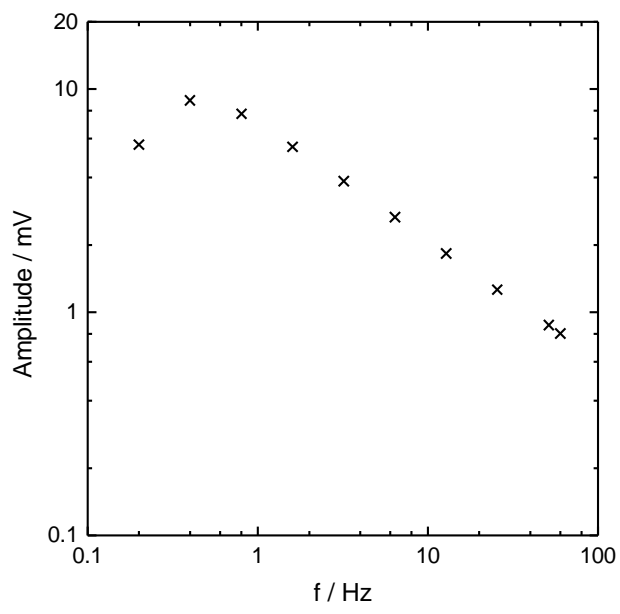

(a)

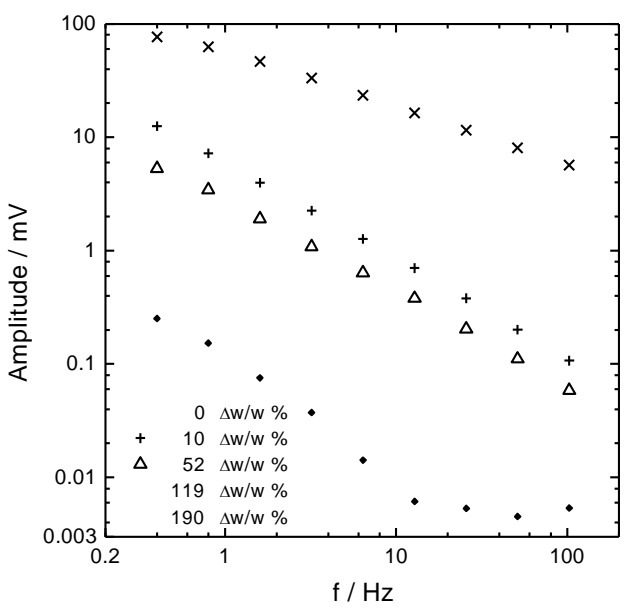

(b)

Figure 1: Measured signal amplitudes for (a) artificial lime sand-stone for both the dry ( $\mathbf{a}$ ) and a wet (a) state, and (b) for the polyamide surface of a two-yarns/stitch fabric for different moisture content. 


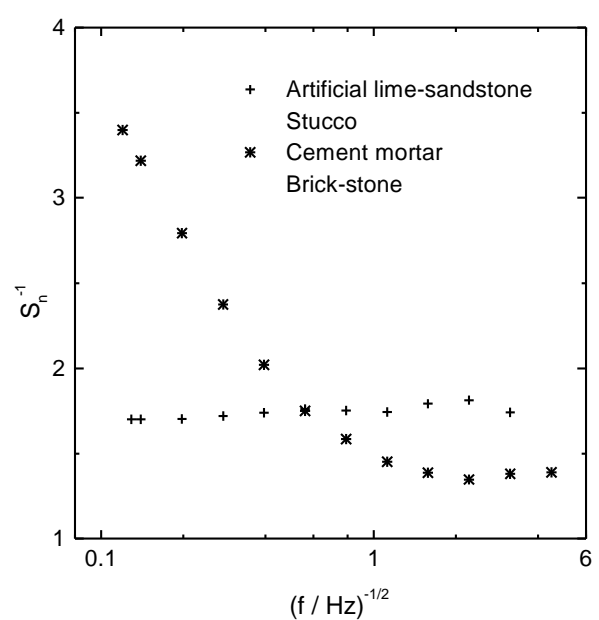

Figure 2: Moisture depth profiles of different construction materials with constant moisture content of $12 \%$ obtained by inverse normalization with the corresponding signals for the dry samples: artificial lime-sandstone $\left({ }^{*}\right)$, stucco $(\Delta)$, cement mortar (o), and brickstone (ם).

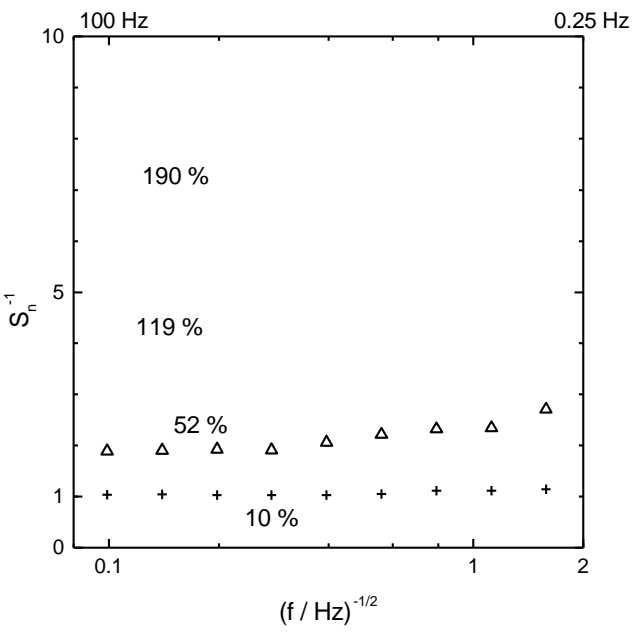

(a)

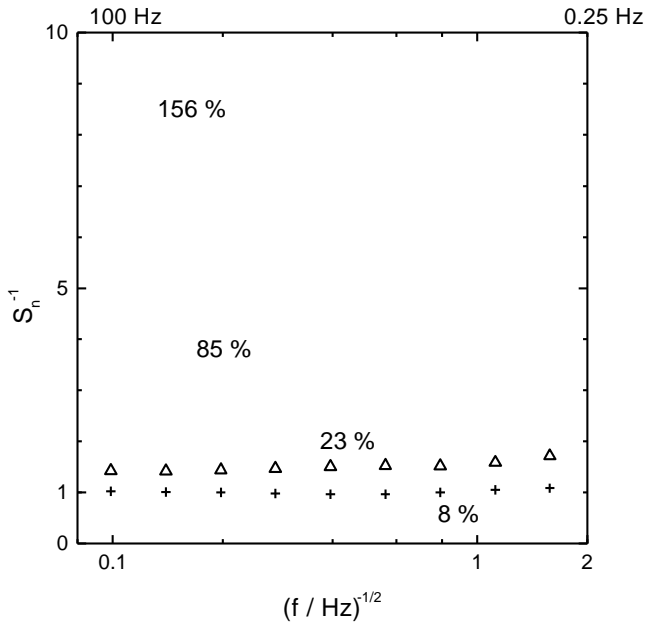

(b)

Figure 3: Moisture depth profiles obtained during the drying of a two-yarns (cotton, polyamide) per stitch fabric measured from (a) the polyamide and (b) the cotton side.

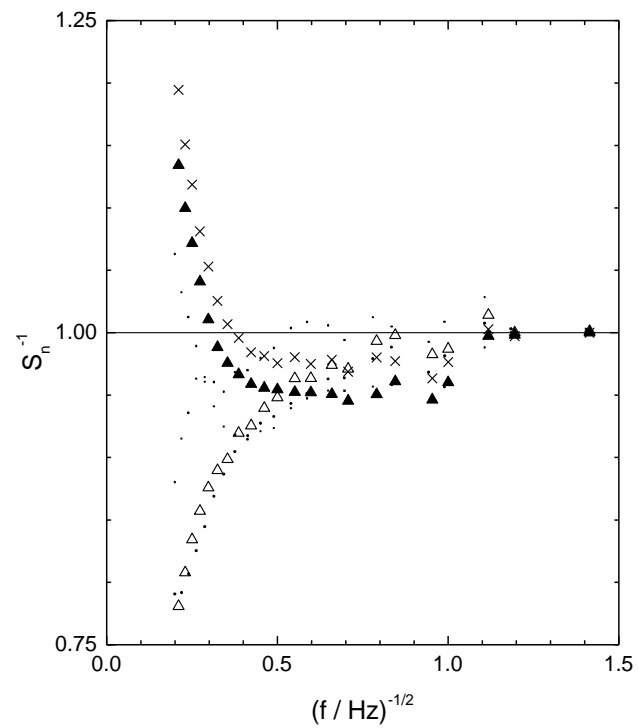

Figure 4: Relative progress of drying of young Gouda cheese observed by comparing the inversely normalized amplitudes obtained at 15

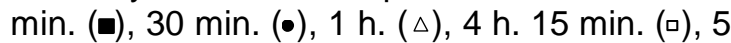
h. (x) and 5 h. 20 min. ( $\Delta)$, with respect to the signal amplitude of the fresh surface cut. 
http://dx.doi.org/10.21611/qirt.2000.041

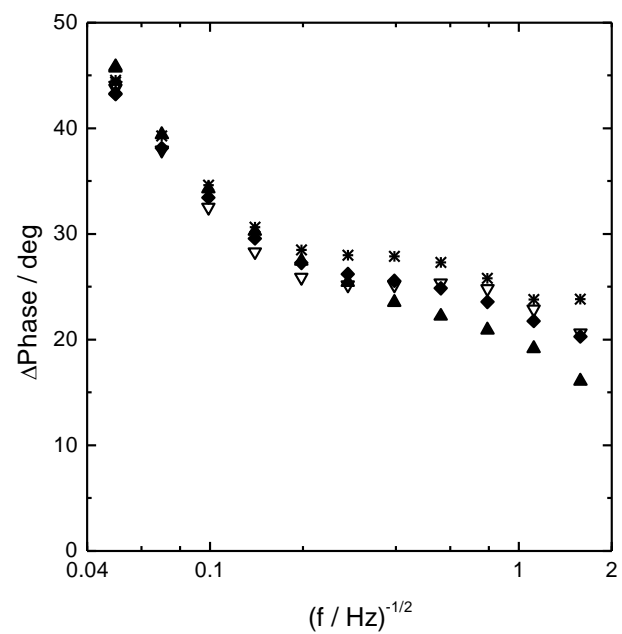

(a)

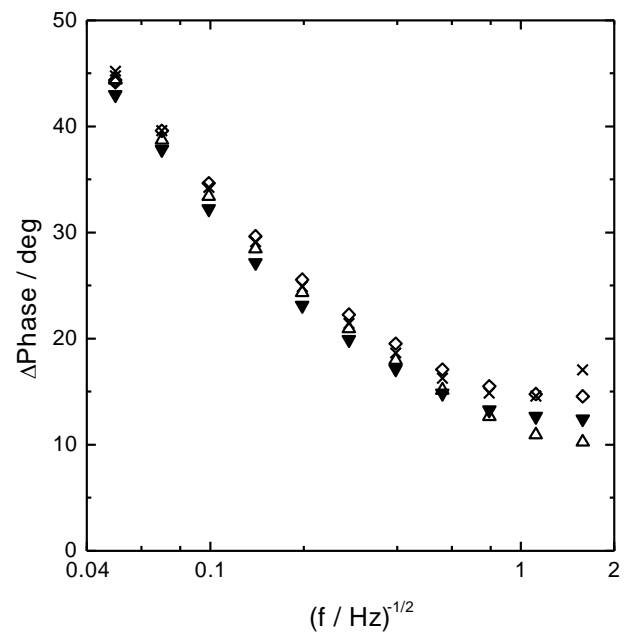

(b)

Figure 5: Relative progress of drying/ripening of German "Rotwurst" observed by comparing the

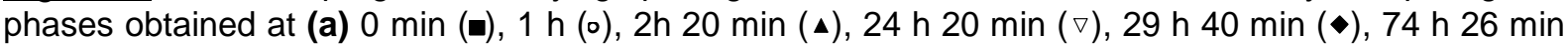
(*) for a thin transparent skin region, and (b) at $30 \mathrm{~min}(\mathrm{(}), 2 \mathrm{~h} \mathrm{( \bullet ),} 2 \mathrm{~h} 40 \mathrm{~min}(\Delta), 25 \mathrm{~h} 45 \mathrm{~min}(\mathbf{v}), 31 \mathrm{~h}$ $(\diamond), 75 \mathrm{~h} 45 \mathrm{~min}(*)$ for a relatively thick white skin region respectively, with respect to the phases measured for an opaque smooth solid (glassy Carbon). 